\title{
LA CONSTITUCIÓN DE SUJETOS POLÍTICOS EN EL MOVIMIENTO ESTUDIANTIL COLOMBIANO
}

\section{THE CONSTITUTION OF POLITICAL SUBJECTS IN THE COLOMBIAN STUDENT MOVEMENT}

\section{Margarita Rosa Vargas Torres}

Universidad Distrital Francisco José de Caldas, Bogotá, Colombia mrvargast69@gmail.com

Resumen: Este artículo tiene por objeto establecer la revisión histórica de lo que ha sido el movimiento estudiantil desde los años 60 hasta la actualidad con el fin de dar elementos para pensar los cambios en las formas de hacer y entender la política que presentan los sujetos que lo integran en la actualidad. En este recorrido se resaltan los aspectos contradictorios y la heterogeneidad en la composición del movimiento estudiantil junto a la discusión entre lo gremial y lo político, sus lógicas democráticas y/o asamblearias; estas últimas se mueven entre el paradigma de la representación o formas de democracia participativa. Se concluye que los estudiantes que integran el movimiento estudiantil han mantenido sus demandas históricamente, así como su lucha por la unidad y la disputa por los sentidos que configuran su identidad y subjetividad. Ellos recientemente despliegan repertorios de lucha novedosos en cuanto a lo comunicativo y lo político.

Abstract: This article aims to establish the historical review of what the student movement has been from the 60s to the present in order to give elements to think about the changes in the forms in which they exercise and understand politics, presented by the subjects that constitute it today. In this journey, the contradictory aspects and the heterogeneity in the composition of the student movement are highlighted along with the discussion between the reivindicative and the political dimensions, its democratic and / or assembly-like logics; the latter move between the paradigm of representation or forms of participatory democracy. It is concluded that the students that compose the student movement have maintained their demands historically, as well as their struggle for unity and the dispute for the senses that shape their identity and subjectivity. They recently display new repertoires of struggle in terms of communication and politics. 


\section{l. Introducción}

La investigación acerca del movimiento estudiantil plantea varios desafíos para los Estudios Sociales dados los cambios epistemológicos, teóricos, históricos y tecnológicos que demandan nuevas formas de ver y de conceptualizar a los actores políticos. El estudio de la historicidad de los sujetos es fundamental para comprender lo que ha determinado a ese sujeto, su pasado, su presente y cómo concibe su futuro, su consciencia histórica, sus subjetividades, imaginarios y representaciones. Las formas de ser sujeto han variado y con ellas las prácticas sociales, culturales y comunicacionales de los jóvenes universitarios, así como la forma en que construye su memoria colectiva. De esta manera, investigar la historicidad, memoria y maneras de significar sus experiencias, las formas como los estudiantes universitarios construyen sus proyectos y cómo esto ha sido visto en la historiografía reciente es de vital importancia.

Los movimientos sociales y en particular el movimiento estudiantil entendido como un movimiento social, en su accionar político generan unas redes sociales y organizativas latentes (difusas) o visibles (concentradas) según el momento de desarrollo de la protesta. (Vommaro, 2017, pp. 119-120). Esas redes se manifiestan como vínculos de proximidad, afinidad, vínculos que no necesariamente son políticos, sino que podrían denominarse como pre-políticos, de amistad o cercanía solidaria y que para el caso del movimiento estudiantil constituyen su Memoria Larga.

Mauricio Archila (2001, pp. 16-42), plantea que para poder hablar de movimiento social tiene que haber una continuidad en las luchas, una duración en el tiempo, una estabilidad en cuanto a la construcción de un sistema ético y valorativo. Aunque estas no son características que se encuentren con claridad en el movimiento estudiantil porque al contrario éste es discontinuo; una investigadora boliviana, Silvia Rivera (1986) citada por Aguilera (2014, pp. 9-10), ha acuñado un concepto que bien puede asumirse para hablar del movimiento estudiantil, éste es el concepto de Memoria Larga. Según Aguilera, a pesar de la discontinuidad de las luchas, sí hay un sistema de acción, ético y valorativo del movimiento estudiantil que se reactiva y se resignifica con la acción colectiva. La Memoria Larga del Movimiento Estudiantil como concepto permite ubicar "permanencias, interrupciones y latencias" precisamente en su lucha histórica por la autonomía, por la educación pública, gratuita y de calidad; y por su vinculación a las izquierdas y a las luchas populares más allá de lo estudiantil.

Aunque en el movimiento estudiantil no hay continuidad, algunas de sus demandas no han cambiado y se mantienen nexos políticos que permiten el hecho de que en la subjetividad colectiva de los estudiantes, sean constitutivos los discursos de la educación popular, de la educación como derecho, que sientan como suyo el Programa Mínimo de los Estudiantes Colombianos de 1971 al igual que el Manifiesto de Córdoba de 1918 y que conmemoren las fechas en las que han muerto sus mártires estudiantiles a través de acciones colectivas, grafittis, murales y conferencias, entre otros. Esta memoria larga es alimentada y se mantiene viva, en gran medida gracias a los líderes y activistas de los partidos políticos de izquierda que plantean una agenda que logra aglutinar a las diferentes capas de los estudiantes que expresan su heterogeneidad como estudiantes independientes, indiferentes, pertenecientes a 
colectivos, grupos académicos, parches (o grupos de amigos) y demás. A través de la historia del movimiento estudiantil, estos activistas convocan, aglutinan, avivan la lucha, controvierten y aportan en la generación de memoria y de movimiento.

Comprender las dinámicas del movimiento estudiantil pasa por conocer lo que se ha estudiado acerca de su devenir histórico incluyendo sus formas de organización, comunicación, su capacidad de movilización, estrategias de comunicación, símbolos, vínculos políticos, entre otros. Las características de los estudiantes del siglo XXI en contraste con aquellos que participaron o lideraron el paro de 1971 involucra vislumbrar las diferencias, por ejemplo, el estudiante de 1971, pensaba en la toma del poder y en la viabilidad de la lucha armada, el liderazgo lo tenían las organizaciones históricas del estudiantado colombiano como son la JUPA, la JUCO, los trotskistas, entre otras. El de hoy, aunque sigue teniendo influencia izquierdista, parece ser adverso a los partidos políticos y prefiere otras formas de asociación como los colectivos, los grupos de estudio o los parches o grupos de amigos que le den cierta independencia. Sin embargo, la presencia de los partidos de izquierda sigue vigente en agrupaciones del estudiantado como la Asociación Colombiana de Estudiantes Universitarios -ACEU, organización estudiantil asociada al Partido Comunista Colombiano - PC, la Organización Colombiana de Estudiantes - OCE, afiliada al Movimiento Obrero Independiente y Revolucionario- MOIR, la Federación de Estudiantes UniversitariosFEU afiliada a Marcha Patriótica, etc.

El movimiento estudiantil colombiano es heterogéneo, aunque durante mucho tiempo sus analistas intentaron clasificarlo y asociarlo a las clases medias (Caycedo, 1967 citado por Archila pg. 397), su identidad, independientemente de su clase social, ha estado dada por su carácter transitorio, generacional y por su cercanía a las izquierdas partidistas, aunque en sus primeras etapas, periodo anterior al derrocamiento de la dictadura de Rojas Pinilla en 1957, su cercanía fue con los partidos tradicionales.

Desde su consolidación en el periodo posterior a 1957, el Movimiento Estudiantil ha buscado levantar la bandera de la calidad de la educación, para lo cual dentro de su repertorio de luchas se encuentra la actualización de los programas académicos. De igual forma históricamente sus demandas se han centrado en dos grandes ejes: un adecuado financiamiento para la educación pública, es decir gratuidad, universalidad y derecho; y autonomía universitaria lo que implica entre otras cosas, el relevo de las directivas en las Universidades, el fortalecimiento de los Consejos Estudiantiles y el cogobierno con una alta participación de profesores y estudiantes en los órganos directivos de las universidades.

Tradicionalmente, también ha apoyado y se ha vinculado a las luchas de otros sectores de la sociedad, como los sindicatos, los campesinos, los barriales, los magisteriales, etc. Como movimiento intelectual y con su modo de actuar beligerante, se ha ganado un reconocimiento y se ha considerado como motor de otras luchas por lo que también ha sido asociado a la izquierda colombiana.

Este artículo se hace en el marco de mi investigación doctoral acerca de la constitución política de los sujetos sociales participantes en el movimiento estudiantil en el siglo XXI. Presenta una revisión bibliográfica de los estudios producidos a partir de la década del 90 con algunas excepciones de estudios realizados en los 70 s y en los 80 s. 
(Medina, 1983, pp. 7-82; Le Bot, 1986, pp. 71-199; Ocampo, 1979, pp. 19-82).

Los textos analizados están divididos en dos grandes grupos; primero, los que abordan lo político-ideológico-organizativo de la acción colectiva estudiantil en donde las principales discusiones tienen que ver con si el movimiento estudiantil debe responder a problemas estructurales de la sociedad colombiana ubicado en el plano de lo político o si su preocupación debería estar orientada a un repertorio reivindicativo relacionado con problemas de la educación y cuál es el papel de las bases estudiantiles y el de los representantes, líderes o voceros en la toma de decisiones. (Cote, 2009, pp. 413-462; Acevedo, 2011, pp. 221-242; Ruíz, 2002, pp. 115-145; Urrego \& Pardo, 2003, pp. 1-20; Cabrera, 2011, pp. 1-6; Medina, 1983, pp. 7-82; Ocampo, 1979, pp. 19-82). En segundo lugar, los textos que hacen referencia a los estudios que abordan la dimensión subjetiva de los movimientos sociales, incluido del movimiento estudiantil, su lenguaje simbólico, narraciones y discursos, sus marcos valorativos, vida cotidiana y construcción de sentido como disputa por lo social y por lo político. (Muñoz, 2007, pp. 69-88; Reguillo, 2008; Retamozo, 2009, pp. 96-117; Torres, 2009, pp. 63-69; Khomasi, 2010, pp. 98-103; 7-13; Castillo, 2011, pp. 101124; Aguilera, 2014, pp. 182-221, García, 2017, pp.27-33.

\section{La historicidad del movimiento estudiantil: entre lo gremial reivindicativo y lo político estructural}

El periodo de consolidación del movimiento estudiantil se enmarca en la situa- ción mundial de la década del 60 caracterizada por la crisis del bloque soviético y la posterior ruptura chino-soviética, así como por la guerra de Vietnam y el movimiento intelectual de mayo de 1968. En este contexto de "resistencia democrática" y posterior "radicalización contra el bipartidismo" (Archila, 2012, pp. 76-77), desde los años 50 surgen varias organizaciones estudiantiles como la Unión Nacional de Estudiantes Colombiano -UNEC, la Federación Universitaria Nacional -FUN y la Federación de Estudiantes Colombianos -FEC. A finales de la década del 50 y en la década del 60 se consolida el movimiento estudiantil construyendo un imaginario social y simbólico de ser un actor con un perfil rebelde, beligerante y contestatario. En este primer abordaje desde lo político-ideológico, se ubicaría el grupo de estudiosos del movimiento estudiantil y quizás el más representativo de lo que hasta la fecha se ha hecho, que han analizado los hitos históricos del movimiento como son la muerte del estudiante Bravo Pérez en 1929, la de Uriel Gutiérrez en el 54 (Medina, 1983; pp. 7-82; Díaz, 2012, pp. 157-189) el paro de 1971 y su programa mínimo, (Urrego \& Pardo, 2003, pp. 1-12; Ocampo, 1978, pp.19-82; Cabrera, 2011, pp. 1-6). En estos textos se reseña la historia monumental de 1929 y de 1954 en la que los estudiantes asesinados son vistos como símbolos que le han dado identidad al movimiento estudiantil. La conmemoración de su muerte se ha vuelto el momento y lugar para fortalecer los vínculos, reavivar las demandas y congregar alrededor del tropel (o disturbio con pedrea) o de la discusión académica con motivo de la celebración del día del estudiante caído.

De otro lado, la memoria es una disputa por la representación de la realidad e incide de manera importante en la sub- 
jetividad política del estudiante universitario, es decir sus formas de sentir, actuar y pensar la política y lo político. En este caso, la memoria de los estudiantes caídos se ha manipulado según los intereses de los grupos políticos en el poder. Por ejemplo, Bravo Pérez ha sido visto como un héroe revolucionario y parte de la literatura ha construido su memoria como el estudiante que lideró el cuestionamiento en contra del gobierno de Abadía Méndez por considerarlo el responsable de la masacre de las bananeras, sin que ese haya sido exactamente su perfil. (Díaz, 2012, pp. 169-170; Castillo, 2011, pp. 101-124).

Los autores que han estudiado el movimiento estudiantil desde lo político-ideológico, entendido esto, como las afinidades con organizaciones o grupos políticos y las creencias que han tenido los estudiantes colombianos, señalan que las motivaciones de las luchas estudiantiles en los 50s y las actuales no han cambiado. Las reivindicaciones siguen siendo por autonomía, financiación, calidad académica, libertad de cátedra, igualdad y justicia social. Sin embargo, lo que sí ha estado en disputa y continúa siendo un dinamizador de los debates al interior de las organizaciones estudiantiles es el papel que debe jugar lo político y la lucha por el poder en la orientación que se le da a las luchas estudiantiles.

Las discusiones sobre si el movimiento estudiantil debe luchar por acometer transformaciones de la estructura económica, social y política del país o si se debe concentrar en lo puramente reivindicativo o gremial correspondiente a demandas relacionadas con la educación aparecen de manera recurrente en los diferentes estudios (Ocampo, 1978; pp. 19-82; Ruíz Montealegre, 2002, pp.117-145; Urra, 2012; pp. 24-37; Urrego, 2003, pp. 1-20). Ruíz Montealegre demuestra en su investigación que en el periodo que él estudia (1954-1966), la lucha política del movimiento estudiantil como lucha por el poder para ejercerlo, o la lucha gremial reivindicativa alrededor de temas relacionados con la cultura y la educación fue la discusión central y lo que marcó los auges y ocasos del movimiento estudiantil. Según el autor, cuando la primera opción ganó preeminencia en el movimiento estudiantil, éste se debilitó como ocurrió a mediados de los 60 s con el ingreso de numerosos activistas a las filas del ELN y del EPL principalmente.

Es de anotar que esta discusión continúa vigente en el siglo XXI, a juzgar por lo reportado en el estudio de Aguilera (2014, pp. 191-194) sobre las discusiones políticas en el paro del 2007 contra el Plan de Desarrollo de Álvaro Uribe, o el dirigido por la Mesa Amplia Nacional Estudiantil -MANE en el 2011 en el que uno de sus dirigentes, Sebastián Pérez (2012, p. 48) manifiesta que una de las tensiones del movimiento estuvo dada por la discusión entre lo gremial y lo político que el adjetiva como "antropofagia política". Este fenómeno según Pérez, no permitió "unidad de sentido" en cuanto a la idea de país y terminó erosionando a la MANE. También Aguilera, señala que otro motivo de tensión es la de aquellos estudiantes independientes que a su manera también defienden unos intereses políticos particulares. Por último, Pérez reseña la tendencia al "estrategismo" que tiene que ver con la intencionalidad de hacer cambios estructurales de la sociedad colombiana.

De otro lado, la lucha por el cogobierno protagonizada por los estudiantes en 1971 también se considera un hito histórico que ha recibido atención de los investigadores interesados en el movimiento estudiantil. Al respecto, Aceve- 
do \& Correa, 2014; pp. 4-55; Urrego \& Pardo, 2003, pp. 1-12 y Cabrera, 2011, ven en este paro uno de los movimientos más masivos y beligerantes de rebeldía juvenil y plantean que fue un dinamizador de luchas posteriores. Según los autores, los estudiantes despiertan simpatías en diversos sectores de la población por su temeridad al enfrentarse al Estado o a la policía, cuando alguna medida estatal afecta el bienestar de la comunidad.

En el periodo de principios de los 70 , esto se evidencia en las manifestaciones de los estudiantes frente al alza de transporte, por ejemplo. Esa simpatía se vio reflejada en el paro del 71, que tuvo como antecedente inmediato la represión de la policía del 26 de febrero de 1971 con un saldo de alrededor de veinte muertes, entre ellos un estudiante. Andrés Caicedo, en el Atravesado citado por Acevedo \& Correa (2014) ilustra la beligerancia del momento del paro con gran fuerza expresiva:

El 26 de febrero prendimos la ciudad de la Quince para arriba, la tropa en todas partes, vi matar muchachos a bala, niñas a bolillo, a Guillermito Tejada lo mataron a culata, eso no se olvida. Que di piedra y me contestaron con metralla. Que cuando hubo que correr corrí como nadie en Cali. Que no hay caso, mi conciencia es la tranquilidad en pasta, por eso soy yo el que siempre tira la primera piedra. p. 131.

El paro del 71 se origina por el sentimiento antiimperialista en contra del Plan Atcon, el Plan Básico, las políticas de fundaciones extranjeras como la Universidad de California, el auge de luchas revolucionarias, los discursos marxistas-leninistasmaoístas que imperaban en la época, la masiva movilización que rechazaba el estado de sitio, entre otras razones. Sin embargo, al igual que en el periodo contemporáneo, todo el proceso del paro se vio marcado por la lucha entre lo gremial y lo político estructural, quienes no apoyaban el cogobierno y preferían un cambio social radical y quienes consideraban que la lucha principal del estudiantado era por el cogobierno.

El paro logró por unos meses el cambio en la composición del gobierno universitario en la Universidad Nacional y en la de Antioquia, en medio de esta lucha, se construyó el Programa Mínimo de los Estudiantes Colombianos que ha inspirado movimientos estudiantiles posteriores, como el de la MANE del 2011.

Los estudiantes colombianos en 1971 consignaron en el Programa Mínimo su demanda por la independencia de la universidad frente al gobierno y por la vinculación de la universidad a la sociedad. Así mismo, en su lucha por la autonomía buscaban sacar a los gremios y a la Iglesia de los órganos de gobierno de las universidades y a cambio lograr mayor participación de estudiantes y profesores en los mismos. De igual forma, exigían al gobierno la reformulación de la financiación de la IES públicas y el pago de la deuda y financiación total de la Universidad Pública. Luchaban por el derecho a la organización de los estudiantes, y por otros métodos de pensamiento como la dialéctica, el marxismo y el materialismo histórico y una ciencia nacional al servicio del pueblo. (Cote, 2009, p. 429).

Este Programa del Movimiento Estudiantil Colombiano logró la unidad del movimiento de manera fugaz, pero pronto se convirtió en una lucha política entre quienes planteaban que no era suficiente desmontar los Consejos Superiores Universitarios de las universidades sino que se necesitaba la Revolución de Nueva Democracia y aquellos que abogaban por 
la revolución socialista argumentando que el cogobierno y la autonomía universitaria eran concepciones liberales burguesas que se daban en el marco del capitalismo. (Le Bot, 1986, pp. 71-199; Cote, 2009, pp. 416-438).

Eso fue lo que sucedió con el cogobierno que no duró sino unos meses tanto en la Universidad Nacional como en la Universidad de Antioquia, dado que no existió la claridad suficiente en el conjunto de las agrupaciones políticas de la época de la importancia de ese instrumento, ni había organización. Los estudiantes colombianos no se han podido recuperar desde hace más de tres décadas de una dolencia, como lo es la ausencia de una organización nacional, sin la cual ningún sector social pueda actuar con eficacia en el terreno de las confrontaciones políticas. (Urrego y Pardo, 2003. p. 13).

Posteriormente al paro de 1971, otro hito histórico de los movimientos sociales fue el paro cívico de 1977, que si bien contó con la participación activa de los estudiantes no fue protagonizado por ellos. Esta manifestación de rebeldía ciudadana ha sido reconocida por los historiadores como una de las más importantes en el siglo XX en Colombia, tal vez después del bogotazo en 1948. Fue convocada por las cuatro centrales sindicales de la época y conjugó el inconformismo por la carestía en el país, el desempleo y la negativa de López Michelsen a negociar los pliegos laborales (Celis, 2007, pp. 1-2). En cuanto a la presencia específica del movimiento estudiantil en el paro del 77, es de destacar la participación del Colegio INEM de Kennedy. En esa época las organizaciones políticas de izquierda como la JUPA, la JUCO, el EPL y el ELN, entre otras, hacían trabajo político en la secundaria. De allí que los discursos de izquierda aunados a un ambiente cultural rico y muy valorado por los jóvenes inemitas de la época provocara la solidaridad de los estudiantes frente a la lucha de los habitantes por servicios públicos, contra el desempleo y la carestía. (Cabrera, 2010, pp. 50-61).

Según Archila (2012, pp. 84-88) del 78 al 80 se da un descenso de las luchas populares probablemente ocasionado por el Estatuto de Seguridad del gobierno de Turbay que prácticamente decretó un estado de sitio permanente y una gran represión a las luchas populares. Es de destacar en los años 80, la lucha estudiantil contra el decreto 80 del 80 que acentuaba la desproporción antidemocrática en los Consejos Superiores Universitarios. Este fue finalmente derogado por la Ley 30 de 1992.

Los años 90 vienen con el protagonismo de los capuchos en las Universidades Públicas y el tropel (disturbio con pedrea) como forma de lucha que era considerada como el último recurso ante la indiferencia de los estudiantes. Los estudiantes que realizan sus performances con el fin de reproducir su ideología forman en gran medida parte de colectivos anarquistas, de la Unión Camilista Revolucionaria, inspirada en Camilo Torres Restrepo y que se expresa con varios grupos en los 90s como los Tercos, Necios y Transformadores (TNT), la Llamarada, Mentes Libertarias, Barricada y el Movimiento Estudiantil Revolucionario (MER). (Vallejo, 2017, pp. 35-43). Es de anotar que estas manifestaciones aunque tenían algún apoyo que les permitía actuar regularmente en el espacio, en general recibía un fuerte rechazo de la mayoría de los estudiantes, que durante las movilizaciones en el paro de la MANE, del 2011 se expresaba con un grito de la multitud que decía "sin violencia" cuando los capuchos hacían presencia.

El contexto en el que surge la MANE y el paro del 2011 coincide con un flujo de las 
luchas populares a nivel mundial, el movimiento Occupy Wall Street, la Primavera Árabe, los Indignados, el movimiento estudiantil en Chile en contra de desajustes en la democracia y en contra del neoliberalismo y su nuevo orden mundial que privatiza lo público que reduce el poder adquisitivo de las personas, endeuda a las familias y cuyo objetivo final es incrementar la ganancia del capital financiero internacional.

El surgimiento de este nuevo orden mundial no se da solamente por la vuelta al capitalismo del bloque soviético y el declarado fin de los metarrelatos, sino también por una crisis del capitalismo que no logra acumular lo deseado y para dar respuesta a su crisis de acumulación, ha impuesto el modelo neoliberal que globaliza los intercambios económicos y culturales. Este modelo, no solamente se apoya en el capital financiero internacional, sino que crea nuevas formas culturales, sociales y políticas para incrementar sus ganancias y sostenerse políticamente.

La globalización debilita la soberanía política de las naciones que empieza a funcionar mediante mecanismos multilaterales o supranacionales a lo que Negri y Hardt (2001, pp.147-195) le han denominado Imperio. Este imperio según los autores, articula lo económico, lo político y lo cultural y mercantiliza todos los aspectos de la vida cotidiana.

En Colombia, la resistencia frente a este orden social y político, se hace en relación con los TLC y con las políticas minero-energéticas por lo que los campesinos protagonizan el paro de trabajadores petroleros en el 2011, los paros agrarios en el 2013 y en el 2014, el paro cafetero también en el 2013 y se presenta el paro nacional estudiantil de la MANE en el 2011 que había tenido un antecedente importante en el paro del 2007. El paro nacional estudiantil del 2007 se da contra el Plan de Desarrollo porque se quería pasar el pasivo pensional de los profesores a las universidades, en él se evidencian ya otras formas de actuar de los estudiantes, otras formas de asociarse o de comprender la asociación, otras formas de actuar y la introducción de lo carnavalesco. El movimiento estudiantil liderado por la MANE se opone a la Reforma a la Ley 30 que plantea la educación como mercancía subordinada al mercado global, así como el fortalecimiento del ICETEX, el ánimo de lucro de la educación superior y la inversión privada en las universidades públicas. Con la presentación de este proyecto de Ley surge la MANE, que se inspira en el Programa Mínimo planteado por los estudiantes en 1971.

En el paro del 2011, los estudiantes hacen un análisis de las oportunidades políticas en el marco de la teoría de la acción colectiva, y dentro de este análisis ubican que con una alcaldía de izquierda como la de Clara López, con el cambio de estilo entre el gobierno de Santos y de Uribe hay condiciones para lanzar el paro (Cruz, 2017, 258-275). Al interior del movimiento estudiantil, en el 2011 y en general, el problema del consenso es de vital importancia, es decir, lograr la unidad política y organizativa es un desafío y lograr hegemonía y consenso de las mayorías estudiantiles, un reto que con mucha dificultad se logra. Un rasgo que podría ser acontecimiento en el 2011, es que en el movimiento estudiantil ya no se acepta el centralismo, las decisiones deben ser tomadas teniendo en cuenta las regiones, las bases y sus diferentes niveles organizativos. En estas nuevas condiciones, el consenso conflictual se puede alcanzar, pero sobre la base de la horizontalidad en la toma de decisiones.

En los paros del 2007 y del 2011, al igual que en Chile 2006 y 2011; los estudiantes 
de las bases no organizadas partidariamente, rechazaron la democracia representativa y prefirieron formas de democracia radical. Esto se expresó en su preferencia por voceros estudiantiles y no representantes estudiantiles y en su negativa a pertenecer a organizaciones políticas. En oposición a esto plantearon otras maneras de hacer política que se evidenciaron en los cambios en el repertorio de acción colectiva, el uso de redes sociales, formas carnavalescas en la movilización, otras solidaridades, democracia radical, etc.

De otro lado, los líderes estudiantiles o muchos de ellos que participaron en el paro nacional estudiantil del 2011, sí pertenecían a organizaciones políticas históricas del Movimiento Estudiantil y con el tiempo, han entrado a participar en la vida política nacional, en el Congreso, algunos son o se han postulado para senadores o representantes a la cámara, son gobernadores, etc., lo cual plantea una idea de la política tradicional, pero también, habla de la complejidad del movimiento estudiantil en el que lo viejo y lo nuevo coexisten y constituyen identidades colectivas plurales y diversas.

A continuación, se ilustra cómo la misma tensión que formó parte de esta sección sobre la política o lo gremial se hace evidente en los aspectos organizativos del movimiento estudiantil.

\section{Lo organizativo: entre la democracia directa, el consenso, la oposición y la unidad}

El movimiento estudiantil despliega un alto nivel organizativo debido a su lucha constante por la unidad, que tal vez es el principal campo de disputa que sortea a su interior y también en relación con el Estado. La organización estudiantil articula dos niveles básicos, el de las bases representadas por asambleas de Consejos de Facultad, de Universidad o de región y las asambleas generales en donde se logran los consensos y se toman las decisiones generales (Aranda,2000, pp. 225250'; Cruz, 2017, pp. 265-268).

En estos escenarios hay un gran esfuerzo por garantizar procedimientos para que los intereses de las mayorías se impongan por encima de los de las organizaciones políticas o de los colectivos organizados que no necesariamente son organizaciones políticas, pero que también representan unos intereses y recientemente se han convertido en un actor importante.

En este terreno, el concepto de hegemonía planteado por Antonio Grasmci, (1975, p. 154, cobra importancia porque corresponde a la capacidad de dirección ético-política especialmente a través del consenso y el control ideológico sin necesidad del uso de la fuerza. Este concepto permite comprender comportamientos de las acciones colectivas contemporáneas en las que emergen nuevas formas de sociabilidad y nuevas formas de tomar decisiones. Aguilera (2014, pp. 182-221) lo ilustra con claridad en su estudio, en particular cuando se refiere a la composición de los actores políticos en la lucha contra el Plan de Desarrollo de Uribe en el 2007 que estaban divididos entre los organizados en partidos políticos, los pertenecientes a colectivos no políticos, pero con un repertorio específico de acción política y los independientes que también participaron activamente en el movimiento.

Sin embargo, ¿quién influía realmente en las decisiones de las asambleas? En primer lugar, las decisiones las "ganan" 
quienes llegan más preparados, quienes llegan con propuestas claras y "saben lo que hay que decir" (Maryorí González, CEM-PRD, CGH. Octubre del 2009, D. F.), aspecto que vincula a la mayoría para legitimar la postura de la minoría. En segundo lugar, muchas de las decisiones de las asambleas las ganan quienes aguantan más, es decir, quienes logran someterse y soportar las largas jornadas (p. 254).

Es claro dentro de los escritos de Cruz (2017, pp. 100-109) que la lucha por el consenso era una forma de mantener la unidad y en este sentido atacar el consenso era atacar la unidad. Según Cruz (2017, pp. 100-109) los grupos estudiantiles denominados "estudiantes de la MANE en disenso" y "Red Nacional de Estudiantes Independientes" hicieron oposición a la "hegemonía nacional de la MANE" y sostuvieron sistemáticamente un discurso antiorganización. Estos estudiantes Ilamados independientes en disenso plantearon una fuerte oposición al liderazgo de la MANE. En la siguiente cita de Cruz, (2013) se explicita con claridad el manejo de la democracia en el paro del 2011:

Las instancias de la MANE y su funcionamiento asambleario fueron desbordados por el acelerado crecimiento de la participación. Las plenarias tuvieron entre 2.000 y 5.000 personas y los Comités Operativos entre 150 y 300. Esto llevó a un debate sobre el manejo de las asambleas y los mecanismos de toma de decisiones. En las asambleas se practica una democracia radical, se eligen moderadores y se discute el orden del día punto a punto hasta alcanzar consensos, si existe disenso la discusión continúa. Los consensos y disensos se recogen en una relatoría aprobada por la plenaria. Sin embargo, también existen relaciones de poder desiguales, acuerdos previos para conformar la mesa moderadora o redactar la relatoría, y una tensión entre el consenso y la mayoría en la toma de decisiones (pp. 59-60).

Aranda (2000) explica que la base de la organización estudiantil

radica en prácticas de democracia directa, además del principio de la división del trabajo y la participación comprometida de todos los integrantes, es decir, se combinan el voluntarismo para determinadas tareas, con la obligatoriedad en otras, lo que permite contar con responsables en todas las tareas y así cubrir los requerimientos del movimiento. (p. 244).

Sin embargo, además de los cambios en la forma en que se toman las decisiones, las dos últimas citas permiten problematizar lo que plantea Retamozo (2009, pp. 104-105), acerca de lo contradictorio en la conformación del movimiento social, en la medida en que manifiesta energía creativa, ilusiones, pero también pesimismos y egoísmos que pueden llegar a reproducir formas caducas de poder como las formas verticales que en ocasiones se presentan en las asambleas de las organizaciones populares, en las que las diferencias en el capital cultural de los participantes concita relaciones jerarquizadas.

\section{I Las experiencias organizativas que sentaron las bases del movimiento estudiantil en Colombia}

Con respecto a experiencias organizativas anteriores, según lo expuesto por Ruíz (2002, pp. 53-86 y por Jiménez (2000, pp. 3-10), la FEC y la UNEC se diferenciaban de la FUN en que para esta última, lo central era la unidad del movimiento estudiantil así como tener la capacidad de ir más allá de las organizaciones políticas. 
Para garantizarlo su estructura organizativa y su unidad, la FUN funcionaba con Consejos Estudiantiles de Facultad lo que permitía que se discutieran y se tuvieran en cuenta los intereses de la base estudiantil. El estudiante común era considerado como elemento cohesionador. Sin embargo, este estudiante de base pasó a segundo plano después del 66 , con la influencia creciente del ELN en el movimiento estudiantil que empezó a estar integrado principalmente por estudiantes militantes de organizaciones políticas. De esta manera, la importancia de los consejos estudiantiles por la respetabilidad, representabilidad y legitimidad ante las autoridades competentes y ante las bases estudiantiles perdió su peso.

Para la FUN, los principios del Movimiento de Córdoba ya no eran suficientes sino que se preocupaba por la construcción de una sociedad más igualitaria en términos económicos y sociales. Personajes como el estudiante Jaime Arenas Reyes, presidente del comité de huelga en el 64 y la presencia de Camilo Torres Restrepo, simbolizan la fuerza que tomó la visión de la política como insurrección revolucionaria según la cual había que fortalecer la conciencia de clase y el espíritu de lucha de los estudiantes universitarios. Ruíz señala que, aunque el grueso del estudiantado no optó por la lucha revolucionaria, varios líderes como Jaime Arenas, quien ingresó al ELN, sí lo hicieron.

En su estudio se evidencia que la fuerza de las organizaciones políticas de izquierda y las guerrilleras, en particular, la irrupción del ELN, las FARC y el EPL en las Universidades minaron y debilitaron la unidad del movimiento estudiantil. Sin embargo, Ruíz, (2002, pp. 213-219) también plantea que el proceso organizativo estudiantil precede a las organizaciones políticas. El autor concluye que, en los 60s, el movimiento estudiantil manejó las tensiones entre universidades públicas y privadas y entre lo puramente gremial y legal y la lucha contra el establecimiento a partir de modelos políticos revolucionarios existentes. Por sus luchas intestinas de carácter ideológico la organización estudiantil no pudo resistir la represión de la protesta estudiantil de Lleras Restrepo lo que marcó el ocaso del movimiento en la década del 60. Esta disyuntiva, es al mismo tiempo una paradoja, porque la lucha por lo gremial que planteaba que no se podría dar sin transformar lo político, es una etapa fuerte del Movimiento Estudiantil. Sin embargo, cuando se plantea dar la lucha política por fuera de la Universidad, el movimiento se fragmenta y se debilita.

En cuanto a experiencias internacionales que han ejercido influencia ya sea por la estructura de oportunidades que hace que el contexto internacional potencie o genere unas condiciones favorables en el plano organizativo encontramos la del movimiento estudiantil en México en 1968 que se dio en defensa de la autonomía universitaria y que terminó con la histórica y fatídica masacre de Tlatelolco acaecida el 2 de octubre de 1968. La estructura organizativa de este movimiento es un ejemplo de democracia en una cultura autoritaria. (Gilabert, 1993, pp. 184-214). El Consejo Nacional de Huelga - $\mathrm{CNH}$, era el máximo órgano representativo y se consideraba un espacio de "resonancia simbólica", con gran reconocimiento en las bases para quienes los integrantes del $\mathrm{CNH}$ eran los constructores de una "nueva socialidad". La otra forma de lucha eran las brigadas que se organizaban en comisiones de propaganda para producir volantes, plegables y 
carteles que se distribuirían en las calles (Calviño, 2012, pp. 61-87).

La estructura del $\mathrm{CNH}$ garantizaba que los líderes respetaran las iniciativas de las bases y no fueran cooptados por el Estado. Tanto la coalición de maestros de enseñanza media y superior pro libertades democráticas como el consejo universitario (máximo órgano de la UNAM) aceptaron y promovieron al CNH como único interlocutor válido ante las autoridades estatales, lo cual incrementó el prestigio de la representación estudiantil. Según Gilabert, (1993, 184-214), luego de un tiempo esta credibilidad disminuyó debido a que se cayó en el mito del Comité Central, se centralizó demasiado y el autoritarismo estatal puso en crisis las formas organizativas participativas con un grado de horizontalidad del movimiento.

El caso de Chile es también interesante porque varios dirigentes y no dirigentes del movimiento atribuyen el éxito del movimiento estudiantil chileno en el 2011 a sus prácticas de organización participativa con una importante horizontalidad. En este movimiento no se hablaba tanto de representantes en términos de la democracia representativa, sino que se introdujeron con fuerza los conceptos "voceros", "bases" y "asambleas" (Urra, 2012, pp. 29-31). Cabe anotar que lo mismo sucedió con la MANE en el paro de 2011 en Colombia y de igual manera esto le dio gran reconocimiento y legitimidad al movimiento.

\section{La subjetividad en los movimientos sociales}

En este apartado se concibe la subjetividad como herramienta para entender la constitución de los Movimientos Sociales como un sistema de acción que implica un sujeto y una subjetividad que disputan "en y por el orden social" (Retamozo, 2009, pp. 96-117). Esta disputa implica revisar la historicidad de los sujetos, más allá de su individualidad o de sus carencias, poder acceder a su conciencia histórica, a su potencialidad en el presente de construir futuro y a la manera como ellos construyen y actúan sobre la realidad caracterizada por un Estado débil que descuida lo social y tiene dificultades para institucionalizar los conflictos.

Aunque la subjetividad en el movimiento estudiantil colombiano es un tema que no se ha trabajado mucho, en este apartado se presenta un rastreo a partir de los estudios sobre las protestas sociales (Archila, 2009, pp. 55-90) y las disertaciones alrededor de los cambios en las concepciones sobre la política y lo político para reconstruir la subjetividad de los movimientos sociales y del movimiento estudiantil contemporáneo en Colombia.

Mauricio Archila (1995, p. 313) ubica al movimiento estudiantil dentro de los nuevos movimientos sociales junto a los movimientos pedagógicos, ecologistas, de mujeres, pacifistas y por los Derechos Humanos. En 1995, señala el autor que el movimiento estudiantil no se ha consolidado como campo de investigación pues sus estudios no han salido del estudiante como actor social y político y no como actor cultural y que, para ese momento, solamente existían capítulos o artículos alrededor de él. Esta situación con corte en el año 2018, no ha cambiado sustancialmente, pero las discusiones sobre subjetividad política en y por fuera del movimiento estudiantil empiezan a abrirse campo para trascender el abordaje de la historia política y social y mirar las formas de sentir, desear y hacer política de los sujetos. (Aguilera, 2014, pp. 21-35; Pie- 
drahita, et al, 2012, pp. 31-44, Alvarado, S. pp. 19-43).

La historicidad propia de la subjetividad de los sectores subalternos provee el marco en el que se construyen las formas de protesta, las demandas, las identidades y los proyectos en un movimiento social. Sobre este último punto y como se ha mencionado atrás, la identidad del Movimiento Estudiantil ha estado dada por su identidad política (con la izquierda) y teórica (con el marxismo). En los 60s y 70s, los estudiantes se veían a sí mismos como un sector social, no clase, no vanguardia, que debía adherirse a los intereses del proletariado y luchar por la transformación de la sociedad con la lucha armada. Esta visión de sí mismos ha cambiado en cuanto a los dos últimos elementos, pero sus demandas asociadas a gratuidad de la educación pública, universalidad, derecho, autonomía y cientificidad siguen siendo las mismas. Cabe preguntarse, cómo estas demandas se actualizan constantemente en la memoria larga del Movimiento Estudiantil.

El movimiento estudiantil ha articulado en su subjetividad que hay injusticia, inequidad y arbitrariedad en la manera como el Estado distribuye los recursos públicos. En esa medida, sus esfuerzos se despliegan a cuestionar el orden social que privatiza, endeuda y empobrece a los estudiantes y a sus familias. Para que se dé la movilización social, esta demanda se manifiesta primero como pedido y luego como reclamo y esto es posible mirarlo históricamente en sus interconexiones con los discursos e idearios nacionales e internacionales recorriendo algunos de los grandes hitos del movimiento estudiantil a nivel nacional e internacional.

Por ejemplo, el Manifiesto Liminar de la Reforma Universitaria de Córdoba de
1918, planteaba cogobierno, autonomía, cambios en la enseñanza y métodos docentes y proyección de la universidad a la sociedad, demandas que si bien se han actualizado en Colombia continúan vigentes y siguen conformando el proyecto utópico del estudiantado colombiano. Con respecto a mayo del 68 , la lucha contra el consumismo, el colonialismo, el autoritarismo y el conservadurismo se materializó en la producción de subjetividad con la noche de las barricadas en el barrio latino que contó con la participación de más de 40.000 estudiantes. Al evocarla los estudiantes entrevistados por Sánchez describen sentimientos de "entusiasmo, alegría, eternidad, se sueña despierto". Las barricadas no tenían utilidad militar, era división, o delimitación del territorio, de la palabra. El movimiento de Córdoba y el de mayo del 68, son constituyentes de la subjetividad colectiva del ME colombiano.

Como plantea Expósito (2017, pp. 394397), la política es una condición de la subjetividad y el sujeto es necesariamente un sujeto político constituido por procesos relacionales y contingentes. Esto se expresa en las demandas que históricamente ha tenido el movimiento estudiantil y que como se ha dicho en este apartado constituyen su subjetividad y que van desde la opción de tomar las armas para transformar la sociedad como sucedió en los años 60 hasta hacer una revolución de las mentalidades como sucedió en la misma década en Francia en mayo de 1968 o luchar por lo reivindicativo propio del plano cultural-educativo que hace parte de la memoria larga del movimiento estudiantil. En este campo se ubica la disputa por los sentidos como lucha hegemónica de los movimientos sociales y del movimiento estudiantil, en particular. 


\section{I La disputa por el sentido en los movimientos sociales}

En la actualidad, las principales luchas políticas y por el poder se dan en el plano de la cultura, en la lucha por las ideas, en la subjetividad o en la manera como los individuos o los colectivos construyen su realidad, la interpretan, la comprenden y actúan sobre ella. (Yudice, 2002, pp. 3643; Jelin, 2002, pp. 44-62). La transformación de los sujetos y el posicionamiento que ellos de manera individual o colectiva hacen, produce nuevos sentidos y significados acerca de lo social, lo político y lo cultural; transforma las identidades y puede tener un significado político y llegar, sin que sea su objetivo, a transformar las estructuras. A través de la función simbólica del lenguaje, de la producción de sentido y del significado, los sujetos como agentes activos posicionan ideas subalternas, manifiestan la voluntad colectiva más allá de lo que orienta el orden dominante, optan por lo utópico (proyecto de futuro), por la producción de imaginarios, representaciones sociales y proyectos colectivos que constituyen identidades y subjetividades (mitos, memorias, mártires, formas de ser, de hacer y de sentir).

El movimiento social a través de su construcción simbólica y sus discursos define nuevos códigos en un juego del lenguaje (De la Garza, 2000, pp. 12-19; Laclau y Mouffe, 1987, pp. 163-176; Torres, 2009, pp. 63-69). En su estudio sobre los "piqueteros" en Argentina, Martín Retamozo propone categorías de los estudios del lenguaje y la retórica que permiten concretar cómo se da esta lucha por el sentido o por el posicionamiento de códigos alternativos. Explica la manera como los movimientos sociales movilizan sentidos en el momento en que constru- yen sus demandas, cuando construyen sus adversarios, en las movilizaciones, en los diferentes espacios de participación; todos ellos son "campos embebidos y condensadores-constructores de subjetividad colectiva" (Retamozo, 2009, pp. 89-131.) Señala, por ejemplo, que sentidos cognitivos se aglomeran con los emotivos y crean formas de razonamiento como la hipergeneralización, la analogía y la equivalencia, la sinécdoque y la metonimia ejemplificados en esta investigación con códigos de sentidos de los participantes en la investigación como "todos los políticos son corruptos" o los vínculos entre clientelismopolítica y corrupción. (p. 199)

El autor ubica a los espacios intersubjetivos del plano organizacional-asociativo como reconfiguradores de la subjetividad individual que también es constitutiva de la subjetividad colectiva. La organización aporta sus códigos normativos, cognitivos y estéticos (su historia, reglamentos, liderazgos y consignas) para la construcción de la idea de un "nosotros" que resignifica y rearticula otros sentidos, como por ejemplo el sentido de la dignidad o de la solidaridad, en oposición al clientelismo o al individualismo como valores tradicionales y caducos propios de la sociedad neoliberal. En esta construcción, es clave que Retamozo, 2009, pp. 219-233) no solamente analiza los sentidos promovidos por los líderes sino también los sentidos de las bases que incorporan sus interpretaciones y trastocan la hegemonía.

Este proceso no se da de manera homogénea sino contradictoria en el que prácticas que reproducen relaciones de poder coexisten con praxis alternativas. Para entender este carácter contradictorio de los movimientos sociales, es interesante ver la lucha interna del movimiento de los desocupados (piqueteros), por desmarcarse o 
resemantizar las relaciones de los actores sociales con las políticas de asistencialismo y clientelismo, así como de otras impulsadas por el gobierno como dispositivo de control del orden social neoliberal con sus ideas de eficiencia y de productividad -significados propios de la dominación social que coexisten y también disputan el sentido. Esta construcción de sentido tiene sus especificidades según el elemento generacional que es un aspecto relevante cuando se habla de movimiento estudiantil y que se revisa a continuación.

\subsection{Lo generacional}

El aspecto generacional siempre ha contado cuando se habla de Movimiento Estudiantil. Aguilera, (2014, pp. 30-32) señala que a lo largo de la historia el cambio generacional ha sido una particularidad de este sector social. En ocasiones el cambio generacional obligado de los estudiantes que cuando se gradúan se van, es considerado un potencial por la renovación en sus prácticas e idearios y en otros casos es visto como una de las razones de su falta de continuidad y fugacidad en la duración de sus organizaciones.

Según los estudiosos de la categoría de Juventud y de Generación, las protestas juveniles contemporáneas no tienen que ver en lo fundamental con las injusticias sino con la imposibilidad de cumplir con sus expectativas. Según ellos la disposición general de los jóvenes estudiantes es trabajar dentro del sistema más que oponerse al mismo. (Muñoz, 2007, pp. 69-88; Reguillo, 2008, 7-13; Castillo, 2011, pp. 101-124). Lo generacional se relaciona con la identidad porque implica la práctica compartida de formas de ver el mundo y de posicionarse frente a él en una circunstancia histórica determinada. Castillo (2011) en particular realizó su investigación entre 2000 y 2006 para estudiar la construcción de ciudadanías juveniles en la vida cotidiana de los estudiantes universitarios de Manizales. En esta investigación las prácticas políticas son pensadas a partir del mundo de la vida cotidiana, a partir de sus sentidos y significados tomando como base los imaginarios colectivos y las representaciones sociales. La configuración del mundo de los jóvenes se analiza en la perspectiva de los movimientos estudiantiles. Se parte de un enfoque sociocultural que tiene en cuenta las categorías de Castoriadis (1975, pp. 3-6) como son lo instituyente, lo instituido y el movimiento.

Castillo hace una breve reseña histórica de las luchas estudiantiles en Colombia iniciando con la inspiración dada por las luchas de Córdoba, Argentina en 1918 cuando los estudiantes universitarios claman por vincular la universidad al pueblo. Se plantea en ese momento que el ser social determina la conciencia social y que desde la universidad se pueden corregir las disfuncionalidades de la sociedad posibilitando así el progreso social. Hace un recorrido por la historia revolucionaria del movimiento estudiantil en los 60s y 70s y concluye con el argumento que se viene desarrollando en cuanto a las transformaciones en las formas de hacer política. Al respecto señala, citando un informe de la CEPAL del 2003 que, en el siglo XXI, los jóvenes se movilizan por la paz, la democracia, la defensa de los derechos humanos, la ecología y otras causas que solo se vinculan indirectamente a su condición de jóvenes. Se distancian de las instituciones públicas (los partidos políticos, el parlamento, la justicia, la policía, etc.) Las tribus juveniles les llaman la atención y 
se constituyen en la forma de expresarse, intensificar sus vivencias personales y encontrar afectividad. Como lo plantea Parra (2017, pp. 536-541), la esperanza en el sujeto no radica solamente en las condiciones externas, materiales sin en la persona en sí misma, en su cuerpo que interactúa con lo real y en el que son fundamentales los afectos.

\subsection{El papel del internet: ¿Aspecto novedoso en el movimiento estudiantil?}

Como lo enuncio en otro escrito, la revolución de la electrónica puede estar ocasionando cambios en la manera de hacer política, las maneras de interactuar virtualmente generan otra mirada frente a la idea de comunidad, de afiliación o de pertenencia. La aparición del internet y de las redes sociales transforma la esfera pública, configura un nuevo espacio público que posibilita otro ejercicio de los derechos, otras maneras de resistir y repeler la injusticia, la exclusión y la marginalidad. (Vargas, 2016, pp. 15-23). Así, las prácticas sociales, comunicacionales y culturales que se generan con la participación de los ciudadanos en la Web configuran nuevas formas de interacción que pueden estar afectando las relaciones de poder y el ejercicio de la ciudadanía:

Corrado y Firestone (1996) plantean que la circulación de la información que internet facilita en tiempo real puede crear esferas públicas en el sentido habermasiano, es decir como comunidades que se congregan para hacer control político al Estado y se constituyen en el filtro/el espacio o el mediador entre los individuos privados y el estado como lo eran desde el siglo XVIII los salones de onces o los cafés, espacios para el debate público (Vargas, 2016, p. 16)
El movimiento estudiantil colombiano, así como el chileno, en el 2011 realizó acciones colectivas que utilizaban diversas aplicaciones tecnológicas como internet, redes sociales, comunicación móvil y mensajería instantánea y fotologs, entre otras, constituyendo así, un "espacio digital de lucha política (García, 2017, pp.27-33). El espacio público digital funcionó como espacio de difusión, disputa y confrontación, gracias a su carácter interactivo libre de dominación, facilitó la participación y resolvió las dificultades técnicas que hasta ese momento hacían imposible que los ciudadanos participaran en política de manera masiva. Aunque dentro del movimiento estudiantil colombiano se observan claras continuidades y latencias en sus luchas, la revolución electrónica puede estar generando unas condiciones para que lo político con su énfasis en lo simbólico, creativo y subjetivo transforme no solamente las maneras de hacer política sino también la naturaleza del sujeto político estudiantil de nivel universitario.

Sin embargo, existen posiciones contrarias a esta afirmación en el sentido de que "las redes sociales en Internet no crean revoluciones ni modifican los regímenes políticos, porque esas son tareas de cada sociedad de acuerdo con su circunstancia." (García, 2017, p. 19). Según esta perspectiva, las redes solamente contribuyen con la propagación de informaciones, convocatorias y de esa manera, convicciones que movilizan a los ciudadanos.

Sin embargo, esta transformación en la identidad política, en el sistema representacional o imaginario de los sujetos no es automático y en ocasiones, aunque el movimiento sea masivo, logre sus demandas y cambie las maneras de ser en el periodo de auge de la movilización, no 
genera modificaciones duraderas en el sistema valorativo, ético o cultural de su comunidad. Este es el caso que evidencia la investigación realizada por Khomasi (2010, pp. 98-103), según la cual, el movimiento liderado por los pingüinos en 2006 no transformó imaginarios o representaciones, no creó una identidad política capaz de ser valorada o integrada en el accionar de sujetos diferentes a quienes la protagonizaron.

Garza (2016, pp. 209-259) informa un caso contrario en el que, en una insurrección popular en Oaxaca en el 2006, no se ganó ninguna demanda, pero quedó en el imaginario social que, por unos días, los sectores subalternos tuvieron el control, desplegaron todas sus capacidades y experimentaron otras formas de vida diferentes a las que impone el neoliberalismo.

Finalmente, es pertinente aludir a la dimensión utópica en los movimientos sociales relacionada con la producción de no-lugares enraizados en el deseo que según Retamozo (2009, pp. 173-175) usualmente se expresan en los nombres que los actores les dan a sus emprendimientos comunitarios que se relacionan con "esperanza" y "sueño de futuro" ( $p$. 174). Como plantea Zemelman (1989, pp. 72-79), la idea del futuro se concretiza en una idea del presente por lo cual el estudio de las relaciones sociales actuales permite comprender las potencialidades de los sujetos sociales estudiados, lo cual aporta metodológicamente al estudio de los movimientos sociales, porque según Zemelman "el presente es la articulación que está en el trasfondo de cada uno de los problemas de análisis particulares que elijamos. Es el significado que tiene hablar del presente como coordenada" p. 76. Según el chileno, "la captación de la articulación, desde sus puntos de mayor activación social, re- presenta lo que entendemos como el paso del pensar histórico al pensar político." p. 78. Así, la agudeza del investigador está en "la reconstrucción de la totalidad sociohistórica de la que es parte en un determinado corte de presente" p. 79.

La posibilidad de elaborar un proyecto es una de las dimensiones fundamentales del sujeto colectivo que participa en el movimiento social. Si no se instituye un horizonte, una proyección desde dónde posicionarse es difícil desarrollar potencialidades de transformación social. Zemelman, (1989, p. 75) ve a los sujetos en clave metodológica porque en su historicidad es posible hacer un doble registro el de lo histórico y el del futuro como proyecto utópico, no como construcción ideológica sino como conciencia histórica que remite a un concepto de realidad más amplio que el definido por la ideología.

\section{A modo de conclusión}

Como se ha visto en la revisión presentada, a lo largo de su historia, el movimiento estudiantil universitario colombiano ha sostenido luchas por la democracia universitaria y por la defensa de la educación como derecho. Estas luchas las ha dado en medio de una gran controversia a su interior ocasionada por las diferentes posiciones políticas de sus participantes en las que la presencia de los partidos políticos de izquierda ha sido una realidad, un detonante y aglutinador de la historicidad del movimiento. El aspecto contradictorio en el movimiento estudiantil hace que se mueva entre dilemas como la lucha por lo gremial concentrado en las demandas educativas y académicas o lo estructural o político con el enfoque de transformación revolucionaria de la sociedad. La perspectiva que se 
presenta del movimiento estudiantil en el siglo XX, lo muestra moviéndose en esos dos ejes sin poderse salir de allí, lo cual plantea la inevitabilidad de la inclusión del discurso político en las discusiones en defensa de la educación, o dicho de otra forma la política como aspecto constitutivo del movimiento estudiantil y la unidad ineludible entre universidad y política.

Otro aspecto contradictorio está dado por la presencia de los partidos políticos de izquierda y la oposición a ello con el argumento de que el movimiento estudiantil debía aglutinar asociaciones estudiantiles para dar luchas civiles y pacíficas sin que sus integrantes tuvieran afiliación política. Se podrían mencionar más aspectos contradictorios como la lucha entre la democracia radical horizontal y directa y la representativa al momento de tomar decisiones. A pesar de defender la primera en muchas ocasiones el movimiento estudiantil caía en la segunda, lo cual se expresa en el manejo asambleario e incluso en el devenir político de muchos de sus líderes (los del 71 y los del 2011, entre otros). También el aspecto contradictorio de los sujetos que luchan en los movimientos sociales se manifiesta en su producción de subjetividad embebida por la subjetividad producida por el neoliberalismo y por las formas caducas de la sociedad tradicional y lo democrático, emancipador y libertario que convoca al movimiento estudiantil.

Para finalizar esta reflexión, es claro que investigar el movimiento estudiantil como movimiento social que implica sujetos y subjetividades debe pasar por el estudio de sus líderes y activistas, pero también y de manera preponderante, por el estudio de las bases estudiantiles conformadas por las mayorías silenciosas, desorganizadas, organizadas en diferentes tipos de colectivos preocupados por lo barrial, los Derechos Humanos, lo ambiental, etc. Quedan señalados algunos elementos que permiten comprender quiénes integraban el movimiento estudiantil en la segunda mitad del siglo XX y a partir de esta heterogeneidad realizar conexiones con el movimiento estudiantil del siglo XXI.

\section{Bibliografía}

Acevedo, A. \& Samacá, G. (2011). "Revolución y cultura en América Latina: el movimiento estudiantil como objeto de estudio en la historiografía colombiana y continental." Revista Memoria y Sociedad. Bogotá, julio-diciembre de 2011.

Acevedo, A. \& Correa, A. (2014). "La movilización estudiantil universitaria del año 2011 en Colombia. Retrospectiva de un síntoma contestatario: 2011-1971." Revista Educación y Desarrollo Social. Universidad Militar Nueva Granada. Bogotá.

Aguilera, A. (2014). Subjetividades políticas en movimiento(s). La defensa de la universidad pública en Colombia y México. UPN- Magisterio Editorial. Bogotá.

Aguilera, O. (2014) Generaciones: Movimientos Juveniles, Políticas de la Identidad y Disputas por la Visibilidad en el Chile Neoliberal. CLACSO. Aguilera, O. (2016). De movidas, movilizaciones y movimientos. Ril Editores. Santiago.

Alvarado S., S. V.; Ospina S., H.; Botero, P. y Muñoz, G. (2008). Las tramas de la subjetividad política y los desafíos a la formación ciudadana en jóvenes. Revista Argentina de Sociología. Vol. 6, Número 11, pp. 19-43. Argentina.

Arendt, H. (2009). La condición humana. Paidós. Buenos Aires.

Aranda, J. (2000). "El Movimiento Estudiantil y la Teoría de los Movimientos Sociales.". UAEM. enero-abril del 2000, Núm. 21, pp. 225-250. México. 
Archila, M. (2012). "El movimiento estudiantil en Colombia. Una mirada histórica. Observatorio Social de América Latina." CLACSO. Buenos Aires.

Archila, M. et al. (2009). Una historia inconclusa. Izquierdas políticas y sociales en Colombia. CINEP. Colombia.

Archila, M. (2005). Idas y venidas, vueltas y revueltas. Protestas sociales en Colombia. 1958-1990. ICANH- CINEP. Colombia. Archila, M. (2001). "Vida, pasión y.... de Ios Movimientos Sociales en Colombia.", en Archila M., Pardo, M. (editores). Movimientos sociales, Estado y democracia en Colombia. CES. UNAL. Colombia.

Archila, M. (1994) Historiografía sobre los movimientos sociales en Colombia. Siglo XX. Universidad Nacional de Colombia. En: vol. Tovar, B. La historia al final del milenio. Ensayos de historiografía colombiana y latinoamericana. Universidad Nacional de Colombia. Bogotá. pp. 251-318.

Cabrera, F. (2011). El Movimiento Estudiantil de 1971, lecciones que deben ser repasadas. Notas Obreras. Net. Bogotá.

Cabrera, L. (2010). La participación de pobladores de Kennedy Central y estudiantes del INEM Francisco de Paula Santander en el Paro Cívico Nacional de 1977. UNAL. Bogotá.

Calviño, E. (2012). La Gráfica del Movimiento Estudiantil de 1968 en México: Una Aproximación desde la Historia del Arte. Centro de Cultura Casa Lamm. México.

Castillo, J. (2011). La configuración de las prácticas políticas en estudiantes universitarios. En: Jóvenes, Culturas y Poderes. Muñoz, G. Bogotá, Siglo del Hombre. ClNEP. pp. 101-124.

Castoriadis, C. (1975). La institución imaginaria de la sociedad. Tusquets Editores. Barcelona.

Celis, L. (2007). El paro cívico de septiembre de 1977. Nuevo Arco Iris. Bogotá.
Cote, Jorge. (2009). "El Movimiento estudiantil de 1971 entre la homogeneidad y la heterogeneidad", en vol. Archila, M. (coord.) Una Historia Inconclusa., CINEP, pp. 413-462. Colombia.

Cruz, E. (2013). La reforma de la educación superior y las protestas estudiantiles en Colombia. Postdata 18, n¹, 2013, pp.51-71. Argentina.

. (2017). Caminando la pala-

bra: Movilizaciones sociales en Colombia (2010-2016). Ediciones desde Abajo, Bogotá.

De la Garza, E. (2000). Subjetividad, cultura y estructura. CLACSO. Argentina.

Díaz, A. (2012). "El 8 de junio y las disputas por la memoria, 1929-1954." Revista Historia y Sociedad. No. 22, Medellín, Colombia, Enero-Junio de 2012, pp. 157189.

Expósito, Julia. (2017). "El pensamiento de Ernesto Laclau. Potencias y limitaciones de su

Crítica a la teoría marxista." Revista Internacional de Pensamiento Político - I Época - Vol. 12 - 2017. España.

García, S. (2017). "Movimientos tecnopolíticos en Latinoamérica. Comparación del \#Yo soy 132 y el movimiento estudiantil chileno." En: Revista Internacional de Pensamiento Político- I Época, Vol. 12 - 2017. España.

Garza, M. (2016). Insurrección, fiesta y construcción de otro mundo en las luchas de la APPO. Oaxaca, 2006-2010. Universidad Autónoma Benito Juárez de Oaxaca. Instituto de Investigaciones Sociológicas Juan Pablos Editor. México.

Gilabert, C. (1993) El hábito de la utopía: Análisis del imaginario sociopolítico en el movimiento estudiantil de México, Editorial Mora. México.

Hardt, M. y Negri, A. (2001). Imperio. Editorial Paidós. Barcelona. 
Jelin, E. (2002). Los trabajos de la memoria. Siglo XXI de España Editores. Madrid.

Jiménez, A. (2000). "Medio siglo de presencia del movimiento estudiantil en la Universidad Pedagógica Nacional." Revista colombiana de educación. No. 40-41.

Khomasi, N. (2010). Identidad Política en el Movimiento de Estudiantes Secundarios de 2006. Memoria para optar al Título Profesional de Sociólogo. Universidad de Chile. Laclau, E. Mouffe, Ch. (1987). Hegemonía y estrategia socialista. Ed. Siglo XXI, Madrid. Le Bot, Y. (1986). Educación e ideología en Colombia. La Carreta, Bogotá.

Medina, C. (1983). Crónicas de violencia: 8 y 9 de junio de 1929 y 1954. Días de lucha estudiantil. Universidad Nacional de Colombia. Bogotá.

Muñoz, G. (2008). ¿ldentidades o subjetividades en construcción? Revista de Ciencias Humanas UTP. No. 37. Diciembre de 2007, Pereira.

Ocampo, J. (1978). Reforma Universitaria 1960-1980. Hacia una educación discriminatoria y antidemocrática. CINEP. Bogotá.

Parra, S. (2017). "El sujeto como esperanza dentro de la encrucijada del nihilismo." Revista Internacional de Pensamiento Político. I Época - Vol 12 - 2017. España.

Pérez, S. (2012). La MANE: tensiones y retos en la construcción del movimiento estudiantil colombiano. UDEA. Antioquia.

Piedrahita, C.; Díaz, A.; Vommaro, P. (2012). Subjetividades políticas: desafíos y debates latinoamericanos. CLACSO. Colombia.

Retamozo, M. (2009). Movimientos sociales. Subjetividad y acción de los trabajadores desocupados en Argentina. FLACSO, México.

(2009). Orden social, subjetividad y acción colectiva. Notas para el estudio de los movimientos sociales. Athenea Digital. No. 16. pp. 95-123. Argentina.
Reguillo, R. (2008). Jóvenes imaginados: La disputa por la representación (contra la esencialización). Revista Punto Cero. Año 13. No. 16. 2008. Bolivia.

Rivera Cusicanqui, S. (1986). Oprimidos pero no vencidos: Luchas del campesinado aymará y qhechwa de Bolivia, 19001980. UNRISD, Ginebra.

Ruíz, M. (2002). Sueños y Realidades. Procesos de Organización estudiantil 1954-1966. Universidad Nacional de Colombia. Editorial Unibiblos. Bogotá.

Torres, A. (2009). "Acción colectiva y subjetividad: Un balance desde los estudios sociales." Revista Folios. Universidad Pedagógica Nacional. Bogotá.

Urra, J. (2012). "La movilización estudiantil chilena en 2011: una cronología" en OSAL. Año XIII, N 31, mayo. CLACSO. Buenos Aires.

Urrego, M. \& Pardo, M. (2003). Primer Congreso Internacional sobre Historia de las Universidades de América y Europa, Universidad de Córdoba - Argentina.

Vallejo, N. (2017). Sombras beligerantes: un estudio sobre las formaciones espaciales de la violencia de los capuchos. Universidad del Rosario. Bogotá.

Vargas, M., Guerrero, C., Quintero, A. (2016). "Ciudadanos mediáticos: ¿Germen de esferas públicas alternativas?" En: Discursos, medios interactivos y políticas educativas. Cap. I. Universidad Distrital Francisco José de Caldas. Bogotá.

Vommaro, P. (2017). Territorio y Resistencias. Configuraciones generacionales y procesos de politización en Argentina. CLACSO. Argentina.

Yudice, G. (2002). El recurso de la cultura: usos de la cultura en la era global. Gedisa. Barcelona.

Zemelman, Hugo. (1989) De la historia a la política. Universidad de las Naciones Unidas y Siglo Veintiuno Editores. México. 